\title{
Strategies for Improving Autonomous Learning Ability of College Students in English Reading in Internet Plus Era
}

\author{
Jia Liu \\ College of Foreign Languages and Literature, Wuhan Donghu University, Wuhan Hubei, 430212, \\ China
}

Key words: Internet plus era, College students, English reading, Autonomous learning ability.

\begin{abstract}
Considering that autonomous learning in English reading under internet conditions can make teaching no longer limited by time and space and then realize interaction of English reading and allow college students to give better play to subjective role of cognition and improve autonomous learning ability, This paper describes the influence of internet plus era on autonomous learning of college students in English reading, interprets autonomous learning model of college students in English reading in internet plus era and puts forwards several strategies for improving autonomous learning ability of college students in English reading in internet plus era.
\end{abstract}

\section{Introduction}

Autonomous English learning ability of college students is one of the important contents of study on English teaching in institutions of higher learning. More and more importance has been attached to it in recent years. Autonomous learning refers to the whole process in which autonomous learners take full responsibility for their learning and master their learning comprehensively in the learner-centered classroom. Learners should determine relevant contents of their learning, learning purpose and objectives to be realized autonomously. The author thinks that autonomous learning should get reasonable guidance of colleges and teachers so that classroom teaching can become a platform to show and promote autonomous learning. In internet plus era, mutual combination of paper, television, broadcasting, network and mobile phone media can be realized with digital means so as to form a brand-new learning pattern. Internet plus era can provide more abundant resources and approaches for autonomous learning of college students as teachers can not only use computer, but also use emerging network media such as Microblog, Wechat and mobile APP for learning and learning contents are no longer limited to traditional time-space concepts such as book, classroom and library. How to use rich and colorful network resources comprehensively to improve the effect of teaching activities and cultivate autonomous consciousness is one of the important issues in current college English teaching.

\section{Influence of internet plus era on autonomous learning of college students in English reading}

Internet plus era provides more abundant resources and space for learning. The use of network for the implementation of autonomous learning can manifest subjective initiative of college students to the greatest extent. English reading teaching is a comprehensive process in which learners understand texts and process relevant information. The use of internet for the implementation of English reading teaching can make English teaching go out of immobilized teaching materials and classroom. Autonomous learning model of college students in English reading in internet plus era has great differences from the teaching-centered teaching mode in traditional sense. It mainly focuses on how to help college students in learning and pays great attention to the establishment of central position of learners and leading role of teachers in teaching. Therefore, how to use network resources more effectively to implement autonomous learning in English reading seems extremely important.

Internet has rich and even endless resources. This knowledge treasure is established according to thinking features of human beings. Therefore, it suits autonomous discovery and exploratory learning of youthful students in colleges and universities. Under this environment, teachers can consider 
individual differences among learners with different learning ability and create a good environment for personalized development and teaching in accordance with students' aptitude. Under this environment, teachers are not simply the only knowledge explainer and students can not only master various teaching contents under positive guidance of teachers, but also use network to obtain relevant knowledge positively, implement selective learning and enhance constant consolidation of current existing relevant knowledge. Under this environment, teachers should become partners of students in autonomous learning and instructors for solving problems. English teachers communicate with students in the process of guidance psychologically, which can further improve teaching atmosphere and provide wider space for autonomous learning of learners. Network is the most ideal teaching assistance means as well as a good medium for the implementation of interactive learning by English teachers. In English reading in internet plus era, discussions on learning can be made completely through network between teachers and students and among students. They can make full use of network technologies and make autonomous learning of English reading have more distinct features. It can provide learning resources closer to the reality such as English newspaper, film and cartoon. Moreover, students can upload their texts, recording and image to network and share with friends and even strangers on the network so as to make autonomous learning of English reading more shared and real-time.

\section{Interpretation of autonomous learning model of college students in English reading in internet plus era}

First, network-based learning data acquisition model. Though English teaching in China always pays great attention to teaching in accordance with the aptitude of students, uniformly printed teaching materials are used in actual teaching process. This immobilized teaching mode severely restricts learning interests of students with different degrees of learning. The development of network and mobile internet media provides new possibility for breaking the restriction. Such media develop into an important supplement in English reading teaching. College students can select various reading materials they consider most appropriate according to their different hobbies, such as economy, military, culture, life and art and contents closely related to their major or hobbies. Teachers should help college students obtain contents they expect to get and guide them in reading methods. Reading in traditional sense mainly involves newspaper and magazine. Extensive use of new mobile media makes mobile electronic reading become the reality. The author thinks that electronic edition of various contents suitable for extracurricular reading of college students such as China Daily, the $21^{\text {st }}$ Century and English Salon can be downloaded with APP for the convenience of electronic reading.

Second, English classroom-based result show model. Autonomous learning is an important method aiming at reallocation of power during knowledge construction and reallocation of participants' role in learning. English reading teaching model with college students as the subject requires college students to become the central link of teaching activities. However, teachers are the central link in reading teaching in traditional sense, who use a reading text as their main teaching material. Their teaching methods are often key and difficult word and sentence translation and syntax analysis etc. Such reading teaching cannot take actual learning status of each learner into account and seems very boring. Moreover, learners can hardly enjoy reading. In fact, learners do not have comprehensive understandings of and interest in English reading. The main objective of English reading teaching is to allow college students to read English newspaper and webpage better. In learning, college students can touch contents above better, select various reading materials they like according to their interest and show objectives of autonomous learning of reading in internet plus era in the classroom.

Three, mutual assistance model based on mutual communication among students. In previous autonomous learning of college students in English reading, a method is often neglected, i.e. mutual assistance among students. Joint learning time of students greatly exceeds the time of contact between teachers and students. Comprehensively using the relationship among students and positively 
encouraging students to learn and check mutually can greatly improve the effect of English reading learning. During extracurricular reading, students can establish English reading interest groups respectively and share their reading materials and learning experience.

\section{Several strategies for improving autonomous learning ability of college students in English reading based on internet plus era}

\section{Improve English reading classroom teaching to improve autonomous reading initiative of learners}

Autonomous learning is a learning activity conducted based on the independence of people. In essence, it is independent learning. Independence can be considered as the soul of autonomous learning of English reading. This requires learners to get rid of inherent learning and thinking mode and avoid depending on others, especially teachers, so as to learn autonomously and independently. Autonomous learning ability is an important foundation of individual learning and professional development. College students can obtain more information according to their learning status and demand. In the constantly developing information times, English teachers should comprehensively change the conception of teaching in traditional sense and emphasize the subjective position of learners so that English reading can actually become internal need and joyful experience of learners. It is necessary to regard English reading as a daily habit, actually help college students to make the most reasonable reading plan and cultivate their independent thinking and ability of solving actual problems. In internet plus era, English teachers should improve classroom teaching means of English reading consciously, develop better English reading circumstances and endeavor to cultivate autonomous reading learning ability of learners. The cultivation of autonomous learning ability cannot be completed in one day. Instead, it is a long-term learning process. Close combination of internet and English reading teaching is a good point of combination.

\section{Establish modern network teaching system so as to form a good autonomous learning chain}

In previous traditional English reading teaching process, English reading materials are often used repeatedly, the updating of which is very slow. Some English teachers pay too much attention to actual accuracy of reading comprehension in reading teaching. Therefore, reading becomes English grammar or translation class. Even if there is after-class practice, it often includes contents such as work recognition and sentence translation. Teaching methods seem to be rigid and boring. In the teaching process, students can hardly select, discover and establish their own English reading knowledge system independently. For English reading comprehension, some students complete corresponding tasks but seldom make their own reading plan or arrange their unique English reading activities reasonably. In internet plus era, the guiding function of English teachers seems extremely important. English reading teaching can use the new platform of network teaching, enhance English reading teaching management and such work as preview and review and then form an autonomous learning chain of English reading with good functions. As English reading is strongly practical, teaching objectives cannot be realized only with teachers' teaching and students' mechanical memory. Network provides a good and new environment of interactive communication for autonomous learning of students.

\section{Enhance English reading guidance so as to improve autonomous reading learning ability of learners}

English reading strategies refer to plans or strategies used in English reading process, various strategies of adjustment and control implemented for reading activities by the subject of English reading in order to guarantee the completion of various reading tasks and the improvement of reading effect, and the process of comprehensive planning and management implemented for various reading elements by readers in order to maximize benefit. English reading strategies have great influence on the implementation of autonomous learning of college students. To cultivate autonomous English 
reading ability of learners, teachers should implement the work of guidance over autonomous reading learning strategies in an ordered, stepped and systematic way and transform reading strategies into actual reading ability of college students. The coming of internet plus era is of great help for the establishment of English learning environment. It is required to implement relevant strategies such as directional selected reading and progressive learning according to specific English reading demands of college students so as to cultivate their special reading abilities such as skimming and skipping reading effectively. For example, many English newspapers have online edition. If reading based on subject, college students can see that a lot of English words are used repeatedly for many times and many words expressing the same or similar meaning occur even in the same report.

\section{Improve English reading course evaluation mechanism so as to enhance self-control ability in English reading under network conditions}

In internet plus era, great importance should be attached to self-control ability of learners. Self-monitoring of college students in English reading learning means that they should consider autonomous English learning activities under implementation as conscious object and conduct continuous planning, examination, evaluation, feedback and adjustment during the implementation of English reading learning activities in order to guarantee the effect of autonomous learning of English reading and realize objectives of English learning. It is required to use comprehensive and objective evaluation to help college students adjust their reading methods effectively, rethink the effect of autonomous reading scientifically and then realize the transformation from passive control by others into active self-control in autonomous learning of English reading.

\section{Conclusion}

On the whole, the improvement of autonomous learning ability of college students in English reading in internet plus era seems more and more important. To implement English teaching under internet plus well, it is required to apply modern educational technologies to autonomous learning of college students in English reading and allow them to use new internet technologies for autonomous learning of English reading. The practice has proved that the implementation of autonomous learning of college students in English reading under internet plus conditions can improve the quality and efficiency of English learning well, enhance learners' awareness and ability of independent learning, promote comprehensive development of college students and well exercise teaching ability of English teachers.

\section{References}

[1] Zhang Dongchang, Pan Weiwei, Chen Weihong. Study on Establishment of English Reading Ability of Chinese Students, Beijing: Intellectual Property Publishing House, 2014.

[2] Chen Shufen. Investigation on Autonomy of Students Majoring in English in English Reading, Journal of Harbin University, 2008 (3).

[3] He Lianzhen, Fu Ying, Fang Fumin, Min Shangchao. Explorations on Cultivation Path of Autonomous Learning Ability of College Students Not Majoring in English in China, Foreign Languages in China, 2011 (5).

[4] Li Peipei. Cultivate Autonomous Learning Ability of Students in College English Reading, Read and Write Journal, 2011 (6).

[5] Gu Qiuping, Gu Xiaoqing, Zhang Sao. Study on Cultivation of Autonomous Learning Ability of Students Majoring in English Based on Network Environment, Exam Week, 2015 (26).

[6] Wang Chunxia. Study on Autonomous English Learning of College Students Based on Network Perspective, College Education, 2013 (12). 